\title{
VISUALIZAÇÃO DA INFORMAÇÃO E MÉTODOS VISUAIS COMO FERRAMENTAS ESTRATÉGICAS PARA O GERENCIAMENTO DE PROJETOS
}

\section{RESUMO}

O gerenciamento de projetos tem possibilitado melhores resultados e vantagem competitiva nas organizações. Os projetos, geralmente, são alimentados por uma vasta quantidade de informações que necessitam ser analisadas e seus significados extraídos para a solução de problemas. Manter um gerenciamento adequado e sistemático não é uma tarefa fácil execução e, muitas vezes, se torna uma barreira. Nessa perspectiva, surgem os métodos visuais e a visualização da informação como ferramentas estratégicas para mitigarem os problemas do gerenciamento de projetos. Por meio de métodos visuais e da visualização, as informações são descobertas e explicitadas, a equipe é coordenada e o volume de informações é tratado substancialmente. O objetivo desse estudo é analisar a influência dos métodos visuais e da visualização da informação nos fatores de gerenciamento de projetos: comunicação, demandas (restrições) e ciclo de vida. Com uma pesquisa descritiva e quantitativa, foi aplicado um questionário semiestruturado com escala do tipo likert, composto por cinco variáveis: ciclo de vida, demandas do projeto, comunicação, visualização da informação e métodos visuais. Foram aplicados 386 questionários a alunos de Pós-Graduação Lato Sensu em Gerenciamento de Projetos, Stricto Sensu em Sistemas de Informações e profissionais da área. Obteve-se 359 respondentes válidos e verificou-se que há o reconhecimento da importância dos métodos visuais e visualização da informação no gerenciamento do projeto, além da existência de relações incipientes.

Palavra-chave: Métodos Visuais. Visualização da Informação. Gerenciamento de Projetos. Demandas do Projeto. Comunicação. Ciclo de vida do Projeto.

\section{VISUALIZATION OF INFORMATION AND VISUAL METHODS AS STRATEGIC TOOLS TO PROJECT MANAGEMENT}

\begin{abstract}
Project management offers better results and competitive possibilities in organizations. Projects are usually fed by a vast amount of information that needs to be analyzed for problem solving. Maintaining proper and systematic management is not a simple task and is often a barrier. From this perspective, visual methods and a vision of information appear as strategic tools to mitigate the problems of project management. Through visual and informational methods, as information is discovered and made explicit, a team of coordination and volume of information are substantially treated. The objective of the study is to analyze the influence of visual methods and information visualization on project management factors: communication, demands (restrictions) and life cycle. Through a descriptive and quantitative research, a semi structured questionnaire with a likert-like scale was applied, composed of five variables: life cycle, project demands, communication, information visualization and visual methods. 386 questionnaires were applied to MBA students in Project Management, graduate students in Information Systems and professionals in the area. Were obtained 359 valid respondents and we verify that there is recognition of the importance of visual methods and information without project management.
\end{abstract}

Keywords: Visual Methods. Information Visualization. Project management. Demands of the Project. Communication. Project Life Cycle.

Fabio José Marques Barbosa ${ }^{1}$ Renata de Souza França ${ }^{2}$

Rodrigo Fonseca e Rodrigues ${ }^{3}$ Fernando Silva Parreiras ${ }^{4}$

\footnotetext{
${ }^{1}$ Mestre em Sistemas de Informação e Gestão do Conhecimento pela Universidade Fumec. Professor da Pós-Graduação da Fumec e da Pontifícia Universidade Católica de Minas gerais - PUC/MG. Brasil. E-mail: fabio71@gmail.com

${ }^{2}$ Doutoranda em Sistemas de Informação e Gestão do Conhecimento pela Universidade FUMEC. Pesquisadora da Universidade Fumec. Brasil. E-mail: profrenatafranca@gmail.com

${ }^{3}$ Doutor em Comunicação e Semiótica pela Pontifícia Universidade Católica de São Paulo - PUC/SP. Professor da Universidade Fumec. Brasil. E-mail: rfonseca@fumec.br

${ }^{4}$ Doutor em Ciência da Computação pelo Universität Koblenz-Landau, Alemanha. Professor da Universidade Fumec. Brasil. E-mail: fernando.parreiras@fumec.br
} 


\section{INTRODUÇÃO}

Movimentos de aumento da concorrência, mudanças tecnológicas e maior exigência por parte dos consumidores, demandam das organizações agilidade, produtividade e alta qualidade. O desenvolvimento de projetos se posiciona entre os processos chave para a competitividade empresarial e tem ganhado o reconhecimento das organizações como alternativa para diferenciação competitiva (Teixeira, Schoenardie, Garcia, Merino, \& Paladini, 2012; Radujković \& Sjekavica, 2017).

O reconhecimento das organizações ocorre pela "adaptabilidade" dos processos do gerenciamento de projetos, que podem ser ajustados conforme necessidades específicas de diferentes setores e níveis de sofisticação (Kerzner, 2011). Porém, a introdução de sofisticadas tecnologias no mercado tem causado mudanças significativas (Pires, 2008).

A onda de ferramentas de gerenciamento de projeto coloca ênfase na colaboração e faz com que as equipes sejam mais produtivas. Essas aplicações caminham lado a lado com modernas práticas de inteligência coletiva e estruturas emergentes que capacitam o gerenciamento de projetos, fazendo com que os projetos transcendam o nível estratégico e impactem diretamente os níveis táticos e operacionais (Kerzner 2011; Lester, 2017).

Comunicar, implementar e monitorar projetos de forma sistemática, orquestrada e de maneira disciplinar, tornam-se um desafio (Lester, 2017) que podem ser mitigados com a utilização da visualização das informações e dos métodos visuais. O uso sistemático de métodos visuais no planejamento estratégico de projetos, possibilita uma variedade de práticas visuais para resolução de problemas (Sindiy, Litomisky, Davidoff, \& Dekens, 2013).

As ferramentas visuais tipicamente incluem, imagens, vídeos, esboços, diagramação, modelagem, prototipagem, entre outros recursos interativos. Essas permitem aos envolvidos tornar a informação transparente e compreensível, auxilia no foco dos processos e explicita o que é necessário (Dusse et al., 2016). Pode-se ainda, utilizar a visualização da informação para fornecimento de dados que gerem informações relevantes do ponto de vista estratégico, por adotar uma metodologia que possibilita a transformação do conhecimento implícito em explícito (Sindiy et al. 2013).

Nessa perspectiva, essa pesquisa tem como objetivo analisar a influência dos métodos visuais e da visualização da informação nos fatores de gerenciamento de projetos: comunicação, demandas (restrições) e ciclo de vida. Busca-se responder a seguinte pergunta: Qual a influência dos métodos visuais e da visualização da informação nos fatores que compõe o gerenciamento de projetos: comunicação, demanda e ciclo de vida?

Esse estudo está estruturado em 6 seções. A primeira apresentou uma introdução do assunto, seguida da segunda seção que apresenta os conceitos centrais e itens da pesquisa. A Seção 3 é composta pelos procedimentos metodológicos utilizados. A Seção 4 realiza uma apresentação dos dados, bem como suas análises e discussões. Seguida da Seção 5, são realizadas as considerações finais. Ao final, apresenta-se as referências utilizadas na construção desse estudo.

\section{GERENCIAMENTO DE PROJETOS}

\subsection{Ciclos de Vida, Demandas e Restrições}

Os projetos são realizados visando alcançar os objetivos do planejamento estratégico de uma organização. Os motivos de sua elaboração podem ocorrer pela demanda do mercado, oportunidade/necessidade estratégica de negócios, solicitação do cliente, avanços tecnológicos ou por requisitos legais (Teixeira et al., 2012). Embora os projetos variem em tamanho e complexidade, com desprendimento de esforço e tempo distintos, podem ser mapeados por meio de uma estrutura genérica de ciclo de vida (PMI, 2013)

Os projetos transcendem o nível estratégico e impactam diretamente nos níveis táticos e operacionais, nos quais são implementados. Implementar e monitorar projetos de forma sistemática, orquestrada e de maneira disciplinar, torna-se um desafio para as organizações. Isso porque o desenvolvimento do plano de gerenciamento estratégico de um projeto é uma atividade iterativa elaborada de forma progressiva no decorrer do ciclo de vida do projeto (Lester, 2017). Segundo Kerzner (2011) o gerenciamento do projeto é composto por métodos de estruturação e técnicas de gestão que possibilitam o controle e a utilização eficiente dos recursos existentes.

O ciclo de vida de um projeto oferece uma estrutura básica para o gerenciamento do projeto, independentemente do trabalho específico envolvido e é constituído por uma série de fases pelas quais um projeto perpassa. As fases são sequenciais e determinadas por: necessidades de gerenciamento e controle das organizações envolvidas no projeto, a natureza do projeto em si e sua área de aplicação (Van den Ende \& Van Marrewijk, 2014; PMI, 2013).

O ciclo de vida de projetos é composto por quatro fases: Início do projeto; Organização e 
preparação; Execução do trabalho do projeto e Encerramento do projeto. O ciclo de vida é independente e não pode ser confundido com os grupos de processos de gerenciamento. Isso porque no grupo de processos as atividades podem ser executadas e ocorrer novamente em cada fase de um projeto bem como em todo o projeto (PMI, 2013; Lester, 2017).

Em sua maioria, as fases do ciclo do projeto são limitadas pelo tempo, com um início e término ou ponto de controle (Van den Ende \& Van Marrewijk, 2014). Nesse sentido e de acordo com os objetivos funcionais ou parciais, as fases do ciclo de vida do projeto podem ser desmembradas considerando os resultados ou entregas intermediárias, ou, até mesmo, disponibilidade financeira. Segundo o PMI (2013), a fase do ciclo de vida atual do projeto deve ser levada em consideração para que haja uma visão de alto nível e um quadro de referência comum que permita comparação de projetos, mesmo que esses não sejam semelhantes.

A estrutura de um ciclo de vida é frequentemente referenciada na comunicação com a alta administração ou outras entidades pouco familiarizadas com os detalhes do projeto. Nessa estrutura, definições relevantes para o projeto são realizadas e as metas, objetivos do projeto, atividades de execução devem ficar explícitas e inteligíveis para todos os envolvidos no projeto (do Valle, 2014; Molena, Rovai, Paes da Rosa \& Plonsk, 2017).

A elaboração progressiva do projeto permite que a equipe de gerenciamento do projeto defina e gerencie o trabalho com um nível maior de detalhes à medida que o projeto evolui, haja vista que circunstâncias específicas do ambiente, das características da equipe e do projeto influenciam no andamento e em seu resultado final (Schneider, Barbosa, Bouzada, \& Gonçalves 2016; Vargas, 2016).
As restrições são os fatores que afetam diretamente o desempenho do projeto e a maneira com que uma atividade será executada. As restrições devem ser identificadas e trabalhadas pelo gerente do projeto e exigem a aplicação de processos e ferramentas de gerenciamento apropriados (Pires, 2008). Os fatores restritivos podem abranger diferentes áreas do projeto, sejam elas escopo, qualidade, cronograma, orçamento, recursos e riscos. Isso porque partes interessadas no projeto podem ter ideias de importâncias divergentes (Holloway, 2016); tem-se a possibilidade de mudança dos requisitos ou objetivos do projeto criarem riscos adicionais (de Melo Paranhos, Bachega, Tavares \& Calife, 2017); A alteração de datas e falta de segmento do cronograma pode alterar os períodos de entrega (Vargas, 2016), entre outros fatores.

Nesse sentido, o processo de comunicação se torna fundamental em um gerenciamento de projetos. A comunicação é o cerne dos fatores determinantes para o sucesso em gerenciamento de projetos, haja vista que projetos são realizados por pessoas que se valem da comunicação para compreender como realizar tarefas, utilizar recursos e cumprir os objetivos estabelecidos (Rocha \& Goldschmidt, 2011; Molena et al, 2017). Comunicação deficiente, falta de clareza nos papéis, excesso de informação, erros de interpretação e entendimento, podem induzir conflitos recorrentes e obstruir o crescimento organizacional (Radujković \& Sjekavica, 2017).

Portanto, a equipe do projeto deve ser capaz de avaliar distintas situações, equilibrar as demandas e manter uma comunicação proativa com as partes interessadas a fim de se entregar um projeto de sucesso. Por meio de referenciais teóricos e da concatenação de autores, tem-se como instrumento de pesquisa para os constructos "Ciclo de Vida do Projeto", "Demandas do Projeto" e "Comunicação" os itens que compõe a tabela 1 .

Tabela1 - Itens do construto "Ciclo De Vida do Projeto", "demandas do projeto - restrições" e "Comunicação".

\begin{tabular}{|l|l|l|l}
\hline & Item & \multicolumn{1}{c}{ Pergunta } & \multicolumn{1}{c}{ Autor (es) } \\
\hline & CV1 & $\begin{array}{l}\text { Na fase de iniciação a missão e os } \\
\text { objetivos dos projetos estão bem definidos. }\end{array}$ & Hollowey (2016) \\
\cline { 2 - 4 } & CV2 & $\begin{array}{l}\text { A fase de planejamento é suficientemente } \\
\text { detalhada. }\end{array}$ & $\begin{array}{l}\text { Van den Ende \& Van Marrewijk } \\
(2014)\end{array}$ \\
\cline { 2 - 4 } $\begin{array}{c}\text { CICLO DE VIDA } \\
\text { DO PROJETO }\end{array}$ & CV3 & $\begin{array}{l}\text { Em geral os projetos realizados pela } \\
\text { instituição, têm alcançado suas metas, em } \\
\text { termos de prazo, custo, qualidade e } \\
\text { satisfação do cliente. }\end{array}$ & $\begin{array}{l}\text { Van den Ende \& Van Marrewijk } \\
(2014)\end{array}$ \\
\cline { 2 - 4 } & CV4 & $\begin{array}{l}\text { Na fase de encerramento do projeto, as } \\
\text { falhas corrigidas são objeto de } \\
\text { aprendizado. }\end{array}$ & $\begin{array}{l}\text { Van den Ende \& Van Marrewijk } \\
\text { (2014) }\end{array}$ \\
\hline & DPR1 & $\begin{array}{l}\text { O escopo é definido adequadamente. } \\
\text { PMI (2013); }\end{array}$ \\
\hline
\end{tabular}




\begin{tabular}{|c|c|c|c|}
\hline & Item & Pergunta & Autor (es) \\
\hline \multirow{5}{*}{$\begin{array}{l}\text { DEMANDAS DO } \\
\text { PROJETO }\end{array}$} & DPR2 & E realizada auditoria de qualidade. & PMI (2013); \\
\hline & DPR3 & O cronograma é cumprido sem atrasos. & PMI (2013); Vargas (2016) \\
\hline & DPR4 & O orçamento é cumprido sem desvios. & PMI (2013); Vargas (2016) \\
\hline & DPR5 & Existem recursos (pessoas) suficientes. & PMI (2013); Vargas (2016) \\
\hline & DPR6 & $\begin{array}{l}\text { É construída uma matriz de riscos com } \\
\text { devidos tratamentos. }\end{array}$ & $\begin{array}{c}\text { PMI (2013); de Melo Paranhos et } \\
\text { al. (2017) }\end{array}$ \\
\hline \multirow[t]{2}{*}{ COMUNICAÇÃO } & $\mathrm{GC} 1$ & $\begin{array}{l}\text { Em geral, os canais de comunicação } \\
\text { utilizados pela equipe de projetos são } \\
\text { eficientes. }\end{array}$ & $\begin{array}{l}\text { Rocha e Goldschmidt (2011); } \\
\text { Molena et al (2017) }\end{array}$ \\
\hline & GC2 & $\begin{array}{l}\text { Em geral, a equipe de projetos se } \\
\text { comunica com sucesso. }\end{array}$ & $\begin{array}{l}\text { Rocha e Goldschmidt (2011); } \\
\text { Molena et al. (2017) }\end{array}$ \\
\hline
\end{tabular}

Fonte: Adaptado de Rocha e Goldschmidt (2011), PMI (2013), Van den Ende \& Van Marrewijk (2014), Hollowey (2016), Vargas (2016), de Melo Paranhos et al. (2017) e Molena et al (2017).

\subsection{Representação Visual em Projetos}

O gerenciamento de um projeto não é tarefa simplória. Unir processos de criação estratégica à sobrecarga de informação e coordenação de múltiplos grupos, dificulta a absorção e a análise de informações, seja pela gerência individual ou coletiva (Lester, 2017). Nesse cenário, estudos empíricos como os de Bauer e Johnson-Laird (1993) e Larkin e Simon (1987), explicitam que a representação visual é superior a representações verbais e pode facilitar a análise de informações representadas por elevado volume.

O processo de visualização em si está relacionado com a transformação de algo abstrato em imagens (mentais ou reais) que possam ser visualizadas pelos seres humanos. O objetivo do processo de visualização é auxiliar no entendimento de um assunto, o qual, sem uma visualização exigiria um esforço maior para ser compreendido (Patterson et al., 2014). O objetivo básico e subjacente a qualquer visualização é constante: a partir de um grande volume de dados extrair o máximo de informação de uma forma rápida, clara e precisa, a partir do uso de metáforas visuais adequadas (Dusse et al, 2016).

A visualização de informação é uma área de aplicação de técnicas de computação gráfica interativas que objetivam auxiliar a análise e a compreensão de um conjunto de dados. É também um campo emergente de pesquisa que se preocupa com a construção de representações visuais de dados abstratos de forma a facilitar o seu entendimento e ajudar na descoberta de informações (Martins Jr., 2016). A visualização da informação permite que pessoas possam processar volume de informação, a partir de suas capacidades inatas de percepção visual, exigindo menor capacidade cognitiva cerebral (Patterson et al, 2014)
Uma boa visualização deve potenciar a clara compreensão da informação e também aumentar a quantidade de informação que é "compreendida" e "apreendida" (Dusse et al., 2016). Isso quer dizer que projetistas, analistas de dados, tomadores de decisão, e outras partes interessadas em um projeto, poderiam concentrar todas as suas atenções cognitivas e perceptivas para o raciocínio analítico ou criativo a partir da visualização das informações, por exemplo (Sibbet, 2013; Reddivari, 2013).

A identificação de valores máximos e mínimos, de desvios, erros, tendências ou intervalos e de "conjuntos" (clusters) de informação de um projeto, são também objetivos presentes na visualização da informação. Os benefícios em se utilizar a visualização da informação na gestão de projetos são amplos, entre eles pode-se destacar: a ampliação das atividades cognitivas dos envolvidos no projeto; melhora no entendimento e aproveitamento do que é exposto; apoio ao processo de aquisição e solidificação de conhecimento por meio da descoberta de informações (Patterson et al., 2014).

Em complementariedade, o uso sistemático de métodos visuais no planejamento estratégico de projetos possibilita a utilização práticas visuais para resolução de problemas. Outra vantagem está no fato das informações se tornarem transparentes e compreensíveis, auxiliando nos processos do projeto e permitindo que se explicite as informações depuradas e necessárias ao andamento do mesmo (Sindiy, Litomisky, Davidoff \& Dekens, 2013). Esses fatores também permitem a compreensão de sistemas complexos, a descoberta de informações encobertas e o suporte no apoio a decisões (Patterson et al., 2014; Dusse et al., 2016).

Pode-se utilizar a visualização da informação para fornecimento de dados que gerem informações relevantes na visão estratégico, por adotar uma 
metodologia que possibilite a transformação do conhecimento implícito em explícito (Sindiy et al., 2013). Vale salientar que, os dados são abstratos e não há, necessariamente, uma representação geométrica. Nesse caso uma imagem é gerada com base nos relacionamentos ou informações que podem se inferidos acerca dos dados.

Por meio de diversos teóricos e modelos, propõem-se os itens do constructo "Visualização da informação" conforme Tabela 2.

Tabela 2 - Itens do construto "Visualização da informação".

\begin{tabular}{|c|c|c|}
\hline Item & Pergunta & Autor (es) \\
\hline VI1 & $\begin{array}{l}\text { A visualização da informação torna a informação explícita e acessível a } \\
\text { todos os envolvidos. }\end{array}$ & $\begin{array}{l}\text { Sibbet, (2013); Sindiy et al. } \\
\text { (2013) }\end{array}$ \\
\hline VI2 & $\begin{array}{l}\text { A visualização da informação possibilita o reenquadramento de pontos } \\
\text { de vista atuais para criação de novas alternativas. }\end{array}$ & Dusse et al., (2016) \\
\hline VI3 & $\begin{array}{l}\text { A visualização da informação facilita a comparação de dados para } \\
\text { definição de uma opção viável. }\end{array}$ & $\begin{array}{l}\text { Patterson et al., (2014); } \\
\text { Dusse et al., (2016) }\end{array}$ \\
\hline VI4 & $\begin{array}{l}\text { A visualização da informação facilita o planejamento, sequenciamento } \\
\text { e interdependências das etapas, para atingimento de metas e objetivos } \\
\text { do projeto. }\end{array}$ & $\begin{array}{l}\text { Patterson et al., (2014); } \\
\text { Dusse et al., (2016) }\end{array}$ \\
\hline VI5 & $\begin{array}{l}\text { As visualizações da informação permitem o acompanhamento do status } \\
\text { do projeto, contribuindo para o engajamento das equipes. }\end{array}$ & $\begin{array}{l}\text { Patterson et al., (2014); } \\
\text { Dusse et al., (2016) }\end{array}$ \\
\hline
\end{tabular}

Fonte: Adaptado de Sibbet (2013), Sindiy et al. (2013), Patterson et al., (2014), Dusse et al., (2016).

\subsection{Desafios estratégicos de projetos e a contribuição dos métodos visuais}

Não é identificado um método visual único que permita analisar os dados com metáforas visuais. Os métodos visuais são representações que fornecem formas de investigar minuciosamente o julgamento gerencial, simplificando e transmitindo ideias acessíveis para debate e modificação.

Tipicamente os métodos incluem imagens, vídeos, esboços, diagramação, modelagem, prototipagem, entre outros recursos interativos. Para identificar o método que se adeque ao projeto, devese considerar: a classificação dos dados e das tarefas, a característica cognitiva do potencial utilizador, o tipo de ênfase visual desejada ao resultado final, entre outros fatores considerados pertinentes (Sibbet, 2013; Reddivari, 2013).

Eppler e Platts (2009) sugerem um framework de visualização da informação composto por quatro etapas: análise; desenvolvimento; planejamento e; implementação, e destacam os métodos visuais substanciais que contribuem no desenvolvimento e implantação de cada etapa. Dentro do framework, os autores sistematizam pontos em que a visualização da informação pode ser aplicada de maneira eficaz e explicitam os métodos visuais que oferecem suporte às atividades do ciclo de vida do projeto (Figura1).

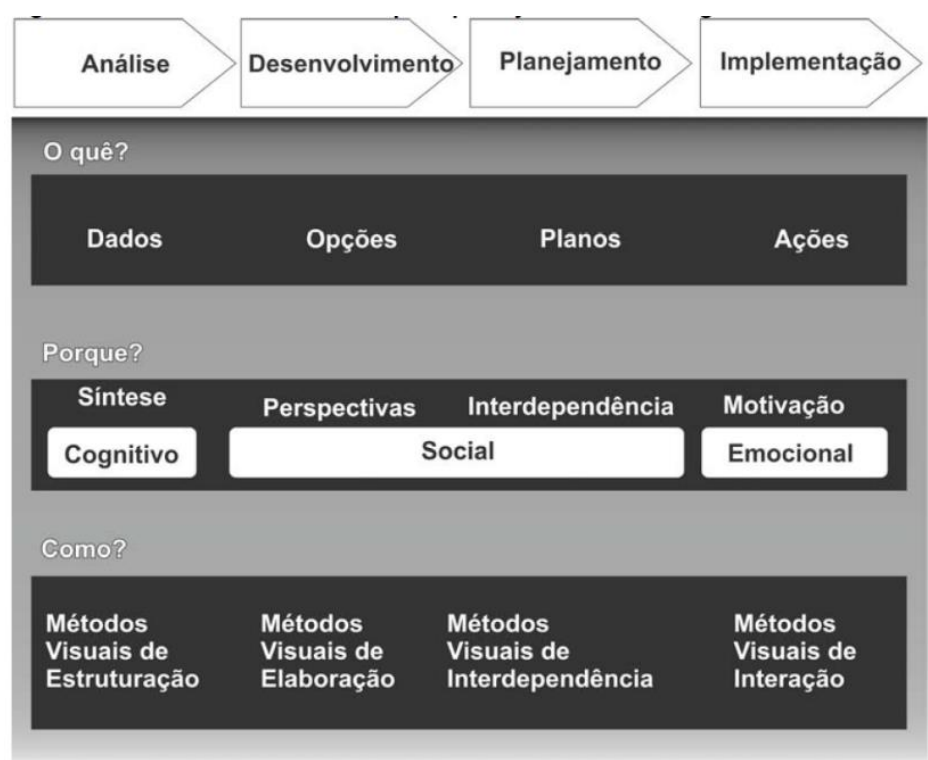

Figura 1 - Framework conceitual para planejamento estratégico visual

Fonte: Eppler e Platts (2009, p 8) 
De acordo com Eppler e Platts (2009) as potenciais contribuições cognitivas dos métodos visuais, incluem: a) o levantamento e síntese de informação, que permite a criação de perspectivas para melhores comparações e a facilidade de recuperação e sequenciamento de informações; b) as contribuições sociais, que incluem a integração de perspectivas e assistência a mútuos entendimentos e apoiam a coordenação entre as pessoas; e, c) as contribuições emocionais, que incluem a criação de envolvimento e engajamento, fornecendo inspiração e comunicação convincente.

A fase de análise do processo do planejamento estratégico de projetos tem como direcionamento o acesso e montagem da estrutura de dados e informação sobre o ambiente interno e externo à empresa, identificando tendências, forças e fraquezas para escolha de métodos e ferramentas que possam gerar diferenciação e geração de valor. Nessa fase, os métodos visuais têm o propósito de representar e sintetizar dados quantitativos e qualitativos.

Tem-se como exemplos de métodos visuais: diagramas, gráficos de linhas, diagrama de sistema, loop, matrizes de 2b2 (BCG, McKinsey, Matriz SWOT), Cinco Forças de Porter, curva-S, gráfico de estratégia e diagrama de produto-mercado (Eppler \& Platts, 2009; Martins Martins Jr., 2016). Os métodos visuais podem fornecer contribuições cognitivas, como, auxilio na composição de dados para elicitação e síntese de informações e obtenção de modelos mentais.

Especificamente a habilidade sintética fornecida pelos modelos visuais, permite aos gerentes processar um maior volume de dados, enquanto evitam sobrecarga de informação, e transmitirem de maneira perceptível os propósitos do projeto (Rose, 2014). Da mesma forma, as informações podem ser estruturadas e convertidas em padrões técnicos, manuais ou modelos, de forma que possam ser customizados pelo o usuário para uma aplicação específica (Banks \& Zeitlyn, 2015).

$\mathrm{Na}$ fase de desenvolvimento do planejamento estratégico de projetos, na qual se busca encontrar e traçar alternativas a serem percorridas, os métodos visuais podem auxiliar na conversão das opiniões das partes interessadas no projeto em potenciais objetivos estratégicos, projetos, atividades e implantações de processos. Os métodos visuais nessa fase permitem o enquadramento de visões atuais e adoção de uma mudança de perspectiva e facilitam comparações sistemática e global de várias opções (Eppler \& Platts, 2009).

Tem-se como exemplos: árvore de decisão, matriz de Ansoff, caixa morfológica, mapa de conhecimento, mapa conceitual, mapa mental, regras de parâmetro, diagramas de influência, business model canvas, design thinking, story telling. Messe sentido, os métodos visuais de elaboração são na maioria utilizados, por fornecer regras e uma estrutura relativamente aberta para elaborar informações, descoberta de padrões e construção do entendimento comum para o desenvolvimento de variadas opções de ação (Eppler \& Platts, 2009; Banks \& Zeitlyn, 2015).

$\mathrm{Na}$ fase do planejamento de estratégias do projeto, o projeto torna-se definido e o objetivo geral é traduzido em objetivos específicos e são formulados planos para atingi-los (Eppler \& Platts, 2009). O benefício dos métodos visuais nessa fase é o de auxiliar e facilitar a comunicação, gerar insights sobre sequências e interdependência de etapas e objetivos, detectar interdependências ou passos das etapas de implementação e que também podem ser úteis na execução. Cronograma, fluxograma, gráfico de Gantt, mapeamento do caminho, diagrama CPM (método caminho crítico), diagrama de PERT, linha de largada, diagrama de loop, mapa de sinergia, são exemplos dos métodos visuais na fase de planejamento.

Métodos visuais de sequenciamento, portanto, são as formas comuns de visualização da informação utilizada nessa fase. Usa-se regras cronológicas, categorias e estruturas gráficas para organizar informações em objetivos e ações; exibese precedentes e interdependências: como cronogramas, regras ou técnicas de elaboração de roteiros; apresenta métodos específicos como: caminho crítico, diagramas de avaliação, evolução do projeto, diagrama de avaliação ou técnicas de monitoramento e controle (Banks \& Zeitlyn, 2015).

$\mathrm{Na}$ fase de implementação dos projetos, métodos visuais que demonstrem os indicadores de desempenho podem fornecer informações valiosas para o planejamento estratégico de projetos. As atividades, processos e resultados apresentam maior relevância quando são visualizados. Um ponto relevante desses métodos visuais é o uso de imagens e símbolos para provocar emoções positivas e motivar a força de trabalho (Eppler \& Platts, 2009; McNely, 2013).

Nessa perspectiva, métodos visuais de interação devem ser usados nesta etapa em busca de fornecer uma interface que permita: capturar, agregar, apresentar e explorar informações. Os exemplos são painel de controle de gestão, metáforas visuais de controle (tais como planos de voo), mapa de estratégia, diagramas de rastreamento, kanban, lean.

Levantou-se teóricos e modelos para propor os itens do constructo "Métodos Visuais" conforme Tabela 3. 
Tabela 3 - Itens do construto "Métodos Visuais".

\begin{tabular}{|c|c|c|}
\hline Item & Pergunta & Autor (es) \\
\hline MVI1 & $\begin{array}{l}\text { Os métodos visuais facilitam o levantamento e síntese do volume } \\
\text { de informações geradas nos projetos. }\end{array}$ & Banks e Zeitlyn (2015) \\
\hline MVI2 & $\begin{array}{l}\text { Os métodos visuais permitem o desenvolvimento de novas } \\
\text { perspectivas e o pensamento divergente. }\end{array}$ & Rose (2014) \\
\hline MVI3 & $\begin{array}{l}\text { Os métodos visuais permitem a comparação de cenários facilitando } \\
\text { a escolha da melhor alternativa. }\end{array}$ & Eppler e Platts (2009) \\
\hline MVI4 & $\begin{array}{l}\text { Os métodos visuais ajudam aos gestores a recordar informações } \\
\text { estratégicas rotineiras. }\end{array}$ & Banks e Zeitlyn (2015) \\
\hline MVI5 & $\begin{array}{l}\text { Os métodos visuais permitem a integração de diferentes } \\
\text { perspectivas equilibrando a participação dos componentes do } \\
\text { projeto. }\end{array}$ & Eppler e Platts (2009) \\
\hline MVI6 & $\begin{array}{l}\text { Os métodos visuais permitem aos Gestores transmitirem instruções } \\
\text { para a compreensão recíproca da equipe. }\end{array}$ & Rose (2014) \\
\hline MVI7 & $\begin{array}{l}\text { Os métodos visuais permitem a integração de equipes } \\
\text { geograficamente dispersas. }\end{array}$ & Eppler e Platts (2009) \\
\hline MVI8 & $\begin{array}{l}\text { Os métodos visuais permitem o envolvimento e engajamento da } \\
\text { equipe nos projetos. }\end{array}$ & Eppler e Platts (2009) \\
\hline MVI9 & $\begin{array}{l}\text { Os métodos visuais permitem a percepção de geração de valor para } \\
\text { a equipe. }\end{array}$ & McNely (2013) \\
\hline MVI10 & $\begin{array}{l}\text { Os métodos visuais permitem ao gestor uma comunicação } \\
\text { convincente com a equipe. }\end{array}$ & $\begin{array}{l}\text { Eppler \& Platts (2009); } \\
\text { McNely (2013) }\end{array}$ \\
\hline
\end{tabular}

Fonte: Adaptado de Eppler \& Platts (2009), McNely (2013), Rose (2014), Banks e Zeitlyn (2015).

\section{MÉTODOS}

A pesquisa caracteriza-se como descritiva, com abordagem quantitativa. Segundo Vergara (2015) pesquisas quantitativas têm por propósito identificar relações entre variáveis por meio da mensuração dos coletados. Para atender ao objetivo dessa pesquisa, que se propõe a analisar a influência dos métodos visuais e da visualização da informação nos fatores de gerenciamento de projetos: comunicação, demandas (restrições conflitantes) e ciclo de vida de projetos, optou-se por realizar a coleta de dados por meio de questionário estruturado do tipo survey, com escala do tipo likert de cinco pontos, variando de 1 , discordo totalmente a 5 , concordo totalmente.

A pesquisa foi realizada com alunos de PósGraduação Lato Sensu em Gerenciamento de Projetos, Stricto Sensu em Sistemas de Informações e profissionais da área, obtendo um total de 386 respondentes. O questionário foi composto por 4 variáveis para caracterização do entrevistado e 27 questões sobre o objeto de estudo, divididas em 5 constructos ("Ciclo de Vida do Gerenciamento de Projetos", "Demandas do Projeto: Restrições", "Comunicação", "Visualização da Informação" e "Métodos visuais").

Excluiu-se 27 indivíduos que não apresentaram respostas para as 27 questões sobre o objeto de estudo, em contrapartida, restaram 359 indivíduos, dos quais realizou-se as análises. Para descrever as variáveis qualitativas de caracterização do entrevistado foram utilizadas frequências absolutas e relativas. Para apresentar e comparar os itens de cada constructo foram utilizados média e desvio padrão e o intervalo percentílico bootstrap de 95\% de confiança para a média.

A escala likert de concordância foi fixada para variar de -1 (Discordo Totalmente) a 1 (Concordo Totalmente), dessa forma, valores médios negativos indicam que os indivíduos tendem a discordar, enquanto que valores positivos indicam que os indivíduos tendem a concordar. A Análise Fatorial Exploratória teve como objetivo a verificação da necessidade de exclusão itens (perguntas) dos constructos que não estivesse contribuindo com a formação do mesmo. Para analisar a qualidade e validade dos constructos foi verificada a dimensionalidade, confiabilidade e validade convergente. Não foi necessária a exclusão de nenhum item.

Utilizou-se as equações estruturais com a abordagem Partial Least Square (PLS) para análise dos dados. A abordagem PLS oferece uma alternativa a abordagem tradicional baseada na estrutura de covariância, Covariance-based Structural Equation Modeling techniques (CBSEM) 
(Monecke \& Leisch, 2012). O software utilizado nas análises foi o R (versão 3.2.4).

Os procedimentos de pesquisa formam realizados com base no trabalho elaborado por Eppler e Platts (2009), no qual os autores descrevem as etapas para o desenvolvimento de um framework. Vale destacar que a escolha do trabalho dos autores como guide line para a pesquisa é devido a afinidade do trabalho a proposta do modelo estrutural.

\section{APRESENTAÇÃO E ANÁLISE DOS RESULTADOS}

Em busca de caracterizar a amostra pesquisada, realizou-se a análise descritiva dos dados. Dos 359 respondentes válidos, $68 \%$ possuem acima de cinco (5) anos de experiência profissional, $19 \%$ ocupam de 3 a 5 anos e $8 \%$ de 1 a 2 anos e apenas. 5\% dos respondentes estão a menos de 1 ano na empresa (Figura 2).

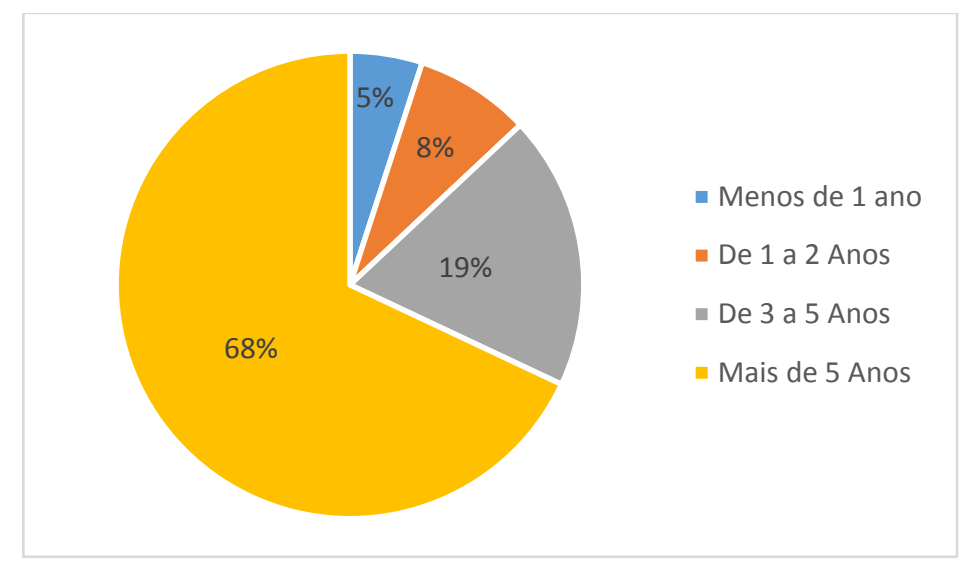

Figura 2 - Tempo de experiência profissional Fonte: Dados da pesquisa

A maioria dos cargos são de gerentes de projetos (13\%) e analistas (38\%). Esse resultado gera confiabilidade nas respostas, haja vista que os respondentes estão inseridos no contexto da pesquisa. Grande parte das empresas (52\%) são de grande porte e possuem como segmento a prestação de serviços $(47 \%)$.

Para descrever as variáveis quantitativas referentes aos constructos, utilizou-se da média, desvio padrão e o intervalo de $95 \%$ de confiança.
Destaca-se que para os respondentes tendem em média a concordar com todas as afirmações do constructo "Ciclo de Vida do Gerenciamento do Projeto". Em uma análise detalhada percebe-se uma maior concordância no item " $\mathrm{Na}$ fase de iniciação a missão e os objetivos dos projetos estão bem definidos (CV1)" quando comparado ao item "A fase de planejamento é suficientemente detalhada (CV2) ", sendo essa a diferença significativa (Tabela 4).

Tabela 4- Medida descritiva constructo "Ciclo de Vida do Projeto"

\begin{tabular}{|c|c|c|c|}
\hline Descrição & Média & $\begin{array}{l}\text { Desvio } \\
\text { Padrão }\end{array}$ & IC- $95 \%$ \\
\hline $\begin{array}{l}\text { CV1 Na fase de iniciação a missão e os objetivos dos projetos estão bem } \\
\text { definidos. }\end{array}$ & 0,25 & 0,59 & $(0,18 ; 0,31)$ \\
\hline CV2 A fase de planejamento é suficientemente detalhada. & 0,11 & 0,60 & $(0,05 ; 0,17)$ \\
\hline $\begin{array}{l}\text { CV3 Em geral os projetos realizados pela instituição, têm alcançado suas } \\
\text { metas, em termos de prazo, custo, qualidade e satisfação do cliente. }\end{array}$ & 0,17 & 0,58 & $(0,11 ; 0,22)$ \\
\hline $\begin{array}{l}\text { CV4 Na fase de encerramento do projeto, as falhas corrigidas são objeto } \\
\text { de aprendizado. }\end{array}$ & 0,26 & 0,66 & $(0,19 ; 0,32)$ \\
\hline
\end{tabular}

Fonte: Dados da pesquisa

O resultado positivo da pesquisa vem enfatizar a importância de se definir objetivos e metas no início de um projeto. Schneider et al.
(2016) e Vargas (2016) enfatizam que a elaboração progressiva do projeto permite que o trabalho seja gerenciado de acordo com as perspectivas 
organizações. Nesse sentido, os objetivos e formas de trabalhos substanciais permitem que as fases do ciclo de vida do projeto sejam desmembradas, planejadas e as disponibilidades de recursos trabalhadas. Por isso, o detalhamento das fases é um fator pontual para o sucesso do projeto (Molena et al. 2017), devido ao fato da mudança de um objetivo poder provocar riscos adicionais (de Melo Paranhos et al., 2017).

Em sequência, no constructo "Demandas do Projeto: Restrições”, os indivíduos tendem em média a concordar com as afirmações "O Escopo é definido adequadamente" e "É realizada auditoria de qualidade" e a discordar da afirmação "É construída uma matriz de riscos com devidos tratamentos", conforme visto na tabela 5. Pires (2008) enfatiza que as restrições afetam diretamente o projeto e a maneira de execução das atividades. Fatores restritivos existem em qualquer projeto, mas devem ser identificados e trabalhados pelo gerente com práticas e ferramentas apropriadas.

Tabela 5 - Medida descritiva constructo "Demandas do Projeto: Restrições"

\begin{tabular}{l|c|l|l}
\hline \multicolumn{1}{c}{ Descrição } & Média & $\begin{array}{c}\text { Desvio } \\
\text { Padrão }\end{array}$ & \multicolumn{1}{c}{ IC- 95\% } \\
\hline DPR1 O escopo é definido adequadamente. & 0,17 & 0,55 & $(0,12 ; 0,23)$ \\
\hline DPR2 É realizada auditoria de qualidade. & 0,12 & 0,66 & $(0,05 ; 0,19)$ \\
\hline DPR3 O cronograma é cumprido sem atrasos. & $-0,04$ & 0,60 & $(-0,10 ; 0,02)$ \\
\hline DPR4 O orçamento é cumprido sem desvios. & 0,01 & 0,60 & $(-0,05 ; 0,07)$ \\
\hline DPR5 Existem recursos (pessoas) suficientes. & 0,03 & 0,61 & $(-0,03 ; 0,10)$ \\
\hline DPR6 É construída uma matriz de riscos com devidos tratamentos. & $-0,13$ & 0,61 & $(-0,19 ;-0,07)$ \\
\hline
\end{tabular}

Fonte: Dados da pesquisa

Observa-se que, nas empresas pesquisadas, o planejamento do projeto com definições de escopo, missões, objetivos são claramente realizadas, inclusive auditadas. Porém, a ausência de uma matriz de riscos com tratamentos devidos pode fazer com que as ações tomadas para mitigarem as restrições e demandas do projeto sejam inadequadas e ineficientes.

Em continuidade, as afirmações "O cronograma é cumprido sem atrasos", "O orçamento é cumprido sem desvios" e "Existem recursos (pessoas) suficientes" os indivíduos não apresentam concordância nem discordância dos itens. De Melo Paranhos et al. (2017) enfatiza que riscos adicionais surgem de fatores como, uma alteração de requisitos, mudança de datas e ou investimentos. Por isso, a possibilidade de se ter planejamentos de soluções melhores se torna um benefício. Esses fatores necessitam ser melhor trabalhados pôr as organizações em questão.

O processo de comunicação é fundamental no desenvolvimento e gerenciamento de um projeto. $\mathrm{Na}$ pesquisa realizada, os respondentes também tendem em média a concordar com ambas as afirmações do constructo "Comunicação", sem diferenças significativas entre os níveis de concordância, conforme tabela 6 .

Tabela 6 - Medida descritiva constructo "Comunicação"

\begin{tabular}{|c|c|c|c|}
\hline Descrição & Média & $\begin{array}{c}\text { Desvio } \\
\text { Padrão }\end{array}$ & IC- $95 \%$ \\
\hline $\begin{array}{l}\text { GC1 Em geral, os canais de comunicação utilizados pela equipe de } \\
\text { projetos são eficientes. }\end{array}$ & 0,18 & 0,61 & $(0,12 ; 0,24)$ \\
\hline GC2 Em geral, a equipe de projetos se comunica com sucesso. & 0,21 & 0,62 & $(0,15 ; 0,27)$ \\
\hline
\end{tabular}

Fonte: Dados da pesquisa

A comunicação é um fator determinante no resultado de um projeto. A comunicação direciona as atividades, os papéis de cada integrante e esclarece os recursos disponíveis no projeto. A inteligibilidade, erros de interpretação e o excesso de informações durante o processo de comunicação podem causar conflitos limitantes a equipe do projeto. Nessa mesma perspectiva, as comunicações deficientes criam restrições aos projetos e obstrui o crescimento organizacional (Rocha \& Goldschmidt, 2011; Molena et al. 2017). Observa-se que as organizações estudadas reconhecem o efeito e a importância de um gerenciamento de comunicação dos projetos.

Em consonância com as afirmações da "Comunicação, os respondentes tendem a concordar 
com todos os itens "Visualização das Informações" (Tabela 7) e "Métodos visuais" (Tabela 8), não havendo diferenças significativas entre os níveis de concordância entre elas, uma vez que os intervalos de confiança se sobrepõem.

Tabela 7- Medida descritiva constructo "Visualização da Informação"

\begin{tabular}{l|c|c|c}
\hline \multicolumn{1}{c|}{ Descrição } & Média & $\begin{array}{c}\text { Desvio } \\
\text { Padrão }\end{array}$ & IC- 95\% \\
\hline $\begin{array}{l}\text { VI1 A visualização da informação torna a informação explícita e } \\
\text { acessível a todos os envolvidos. }\end{array}$ & 0,58 & 0,46 & $(0,53 ; 0,63)$ \\
\hline $\begin{array}{l}\text { VI2 A visualização da informação possibilita o reenquadramento de } \\
\text { pontos de vista atuais para criação de novas alternativas. }\end{array}$ & 0,51 & 0,45 & $(0,46 ; 0,55)$ \\
\hline $\begin{array}{l}\text { VI3 A visualização da informação facilita a comparação de dados para } \\
\text { definição de uma opção viável. }\end{array}$ & 0,52 & 0,47 & $(0,47 ; 0,57)$ \\
\hline $\begin{array}{l}\text { VI4 A visualização da informação facilita o planejamento, } \\
\text { sequenciamento e interdependências das etapas, para atingimento de } \\
\text { metas e objetivos do projeto. }\end{array}$ & 0,50 & 0,47 & $(0,46 ; 0,55)$ \\
\hline $\begin{array}{l}\text { VI5 A visualização da informação permite o acompanhamento do } \\
\text { status do projeto, contribuindo para o engajamento das equipes. }\end{array}$ & 0,55 & 0,47 & $(0,50 ; 0,60)$ \\
\hline
\end{tabular}

Fonte: Dados da pesquisa

Tabela 8 - Medida descritiva constructo "Métodos Visuais"

\begin{tabular}{|c|c|c|c|}
\hline Descrição & Média & $\begin{array}{l}\text { Desvio } \\
\text { Padrão }\end{array}$ & IC- $95 \%$ \\
\hline $\begin{array}{l}\text { MVI1 Os métodos visuais facilitam o levantamento e síntese do } \\
\text { volume de informações geradas nos projetos. }\end{array}$ & 0,49 & 0,47 & $(0,44 ; 0,54)$ \\
\hline $\begin{array}{l}\text { MVI2 Os métodos visuais permitem desenvolvimento de novas } \\
\text { perspectivas e o pensamento divergente. }\end{array}$ & 0,41 & 0,51 & $(0,36 ; 0,46)$ \\
\hline $\begin{array}{l}\text { MVI3 Os métodos visuais permitem a comparação de cenários } \\
\text { facilitando a escolha da melhor alternativa. }\end{array}$ & 0,48 & 0,49 & $(0,44 ; 0,53)$ \\
\hline $\begin{array}{l}\text { MVI4 Os métodos visuais ajudam aos gestores a recordar informações } \\
\text { estratégicas rotineiras. }\end{array}$ & 0,44 & 0,51 & $(0,39 ; 0,49)$ \\
\hline $\begin{array}{l}\text { MVI5 Os métodos visuais permitem a integração de diferentes } \\
\text { perspectivas equilibrando a participação dos componentes do projeto. }\end{array}$ & 0,43 & 0,47 & $(0,38 ; 0,47)$ \\
\hline $\begin{array}{l}\text { MVI6 Os métodos visuais permitem aos Gestores transmitirem } \\
\text { instruções para a compreensão recíproca da equipe. }\end{array}$ & 0,50 & 0,47 & $(0,45 ; 0,55)$ \\
\hline $\begin{array}{l}\text { MVI7 Os métodos visuais permitem a integração de equipes } \\
\text { geograficamente dispersas. }\end{array}$ & 0,40 & 0,50 & $(0,34 ; 0,45)$ \\
\hline $\begin{array}{l}\text { MVI8 Os métodos visuais permitem o envolvimento e engajamento da } \\
\text { equipe nos projetos. }\end{array}$ & 0,47 & 0,46 & $(0,42 ; 0,51)$ \\
\hline $\begin{array}{l}\text { MVI9 Os métodos visuais permitem a percepção de geração de valor } \\
\text { para a equipe. }\end{array}$ & 0,47 & 0,48 & $(0,42 ; 0,52)$ \\
\hline $\begin{array}{l}\text { MVI10 Os métodos visuais permitem ao gestor uma comunicação } \\
\text { convincente com a equipe. }\end{array}$ & 0,49 & 0,48 & $(0,44 ; 0,54)$ \\
\hline
\end{tabular}

Fonte: Dados da pesquisa

A visualização da informação surge para auxiliar no entendimento dos aspectos do projeto que exigiriam esforço maior para serem compreendidos sem esse recurso (Patterson et al., 2014). Reddivari (2013), Sibbet (2013) e Sindiy et al. (2013) salientam que na visualização da informação é utilizada, as informações se tornam evidentes e compreensíveis e é auxilia nos processos do projeto e na solução de problemas. As informações se tornam relevantes e explícitas, de forma a descobrir informações correntes e gerar conhecimentos válidos.

Dentro dessa proposta, encontram-se métodos visuais que possibilitam a análise dos dados e que são contemplados pelo processo de visualização da informação. $O$ resultado dessa pesquisa corrobora as ideias de Eppler e Platts 
(2009), Patterson et al. (2014) e Dusse et al. (2016) demonstrando a importância dos métodos visuais no exame das visões gerenciais e na simplificação e acessibilidade às informações e ideias (Eppler \& Platts, 2009).

Verifica-se que, a partir das respostas dos entrevistados, os métodos visuais são observados como ferramentas de apoio ao gerenciamento de projetos, soluções de problemas e tomadas de decisões gerenciais. Percebe-se também que os benefícios dos métodos visuais estão no levantamento e síntese de informações, criação de perspectivas de comparações, facilidade de recuperação e sequenciamento de informações, entendimento mútuo e valorização da comunicação e integração de ideias e perspectivas (Eppler \& Platts, 2009).

4.1 A influência dos métodos visuais e da visualização da informação no gerenciamento da comunicação, demandas (restrições conflitantes) e ciclo de vida de projetos

Em busca de verificar a influência dos métodos visuais e da visualização da informação foi realizada a análise do modelo estrutural. A figura 3 esboça o modelo estrutural proposto para essa pesquisa, que abrange os constructos "Visualização da Informação", "Métodos Visuais", "Demandas do Projeto", "Ciclo de Vida" e "Comunicação".

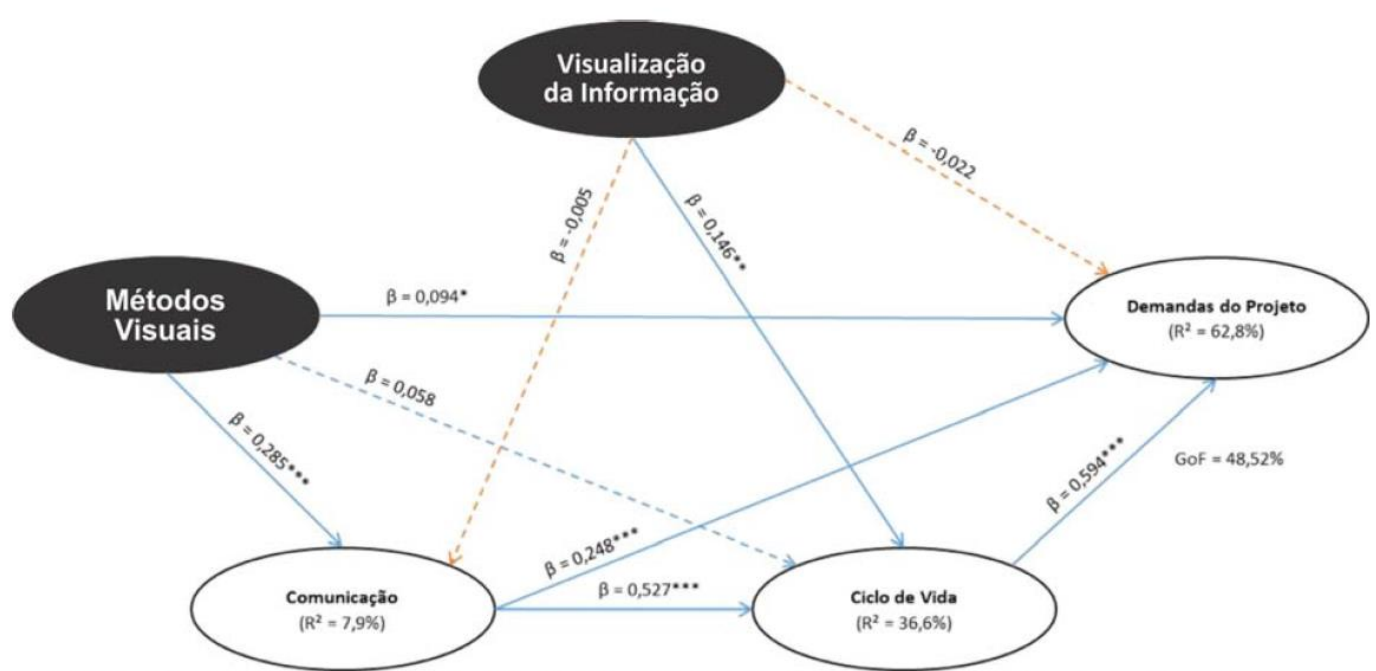

$\left.{ }^{* * *}\right)$ Significativo ao nível de $1 \% ;\left({ }^{* *}\right)$ Significativo ao nível de $5 \% ;\left({ }^{*}\right)$ Significativo ao nível de $10 \%$.

Figura 3 - Ilustração do modelo estrutural

Fonte: dados da pesquisa

Verificou-se que a visualização da informação possui relação direta, positiva e significativa no ciclo de vida do projeto. Isso quer dizer que quanto maior for o apelo à visualização da informação melhor será o ciclo de vida do projeto. Em contrapartida, a influência dos constructos Comunicação e Demandas do Projeto são indiretamente proporcionais.

Utilizar a visualização da informação permite descobertas de informações relevantes do ponto de vista estratégico e possibilita a adoção de uma metodologia que transforme o conhecimento implícito em explícito (McNely 2013; Reddivari, 2013 e Sibbet, 2013). A visualização da informação na gestão de projetos vem como uma ferramenta estratégica que amplia as atividades cognitivas da equipe do projeto, melhorando o entendimento e aproveitamento do que é exposto, e apoia a execução das atividades (Dusse et al. 2016; Patterson et al., 2014).

Avaliando os métodos visuais, identificou-se que há uma influência significativa e positiva desse construto na comunicação. Esse resultado enfatizando a importância dos métodos visuais na eficácia da comunicação em gerenciamento de projetos, haja vista que a utilização inteligente dos métodos visuais adequa os objetivos da comunicação. A mesma situação ocorre com o constructo demandas do projeto, sendo que quanto mais forem utilizados métodos visuais, mais condizentes serão as demandas.

A visualização das atividades, processos e resultados do projeto, junto a utilização dos métodos fornecem contribuições cognitivas que auxiliem na composição dos dados do projeto, na síntese de informações, na facilidade de visualização e na tomada de decisão (Sibbet, 2013; Reddivari et al., 
2013). A exemplificação considerável desses métodos visuais é o uso de imagens e símbolos para provocar emoções positivas e motivar a força de trabalho (Eppler \& Platts, 2009; McNely ,2013).

Porém, chama-se a atenção para a ausência de influência entre os métodos visuais e ciclo de vida do projeto. O PMBOK (2013) salienta que a fase do ciclo de vida deve ser levada em consideração para que haja uma visão de alto nível e um quadro de referência que permita a comparação de projetos. Os métodos visuais são boas referências para apoio nesse processo de comparação e permite uma visualização explícita e sistêmica dos processos e atividades do projeto. A ausência da relação dos construtos pode influenciar nas restrições e riscos dos projetos nas organizações pesquisadas.

Em complementariedade à pesquisa, verificou-se que a comunicação influencia a demanda do projeto e a influência o ciclo de vida do gerenciamento do projeto. Pires (2008) salienta que as demandas de um projeto afetam o projeto, o desempenho do projeto e a forma como a atividades são executadas. Cabe a equipe do projeto avaliar as situações ambientais, equilibrar as demandas e manter uma comunicação proativa com as partes interessadas, a fim de se entregar um projeto com êxito.

\section{CONSIDERAÇÕES FINAIS}

Os métodos visuais e a visualização da informação surgem como ferramentas estratégicas que apoiam o gerenciamento de projetos e mitigam as barreiras e restrições encontradas em seus processos e atividades. Esse estudo buscou, com uma pesquisa quantitativa, analisar a influência dos métodos visuais e da visualização da informação nos fatores de gerenciamento de projetos: comunicação, demandas (restrições) e ciclo de vida. Foram aplicados questionários survey com escalas do tipo Likert de cinco pontos.

Por meio dos resultados e análises da pesquisa, identificou-se que há reconhecimento por parte dos respondentes quanto aos benefícios da utilização dos métodos visuais e da visualização da informação nos resultados do projeto e de seu gerenciamento. Os métodos visuais possibilitam melhorias no desempenho de projetos, nas demandas do projeto e na comunicação. Da mesma forma, identificou-se que, o uso da visualização da informação desempenha um papel relevante no ciclo de vida dos projetos.

Não se pode dizer que há influência nas relações entre visualização da informação e comunicação, visualização da informação e demandas do projeto, e métodos visuais e ciclo de vida do projeto. Dessa forma, essas relações necessitam de atenção e afinco.

Esse estudo conecta dois campos da ciência que apoiam o planejamento estratégico gerencial e no gerenciamento de projetos organizacionais. Porém, salienta-se que os resultados não servem de referência primordial para pesquisas que discorram sobre as temáticas levantadas. Para diferentes organizações e respondentes, podem haver resultados distintos.

Propõem-se como projeto futuro a análise das boas práticas dos métodos visuais utilizadas nas organizações e, ou, uma análise qualitativa da influência dos métodos visuais e visualização da informação nos fatores de gerenciamento de projetos.

\section{REFERÊNCIAS}

Banks, M., \& Zeitlyn, D. (2015). Visual methods in social research (2 ${ }^{a}$ ed.). Los Angeles: Sage.

Bauer, M. I., \& Johnson-Laird, P. N. (1993). How diagrams can improve reasoning. Psychological science, 4(6), 372-378.

de Melo Paranhos, M., Bachega, S. J., Tavares, D. M., \& Calife, N. F. S. (2017). Application of failure mode and effects analysis for risk management of a project. Sistemas \& Gestão, 11(4), 444-454.

do Valle, A. B. (2014). Fundamentos do gerenciamento de projetos $\left(3^{a}\right.$. Ed). Rio de Janeiro: Editora FGV.

Dusse, F., Jr., P. S., Alves, A. T., Novais, R., Vieira, V., \& Mendonça, M. (2016). Information visualization for emergency management: A systematic mapping study. Expert Systems with Applications, 45(1), 424-437.

Eppler, M. J., \& Platts, K. W. (2009). Visual strategizing: the systematic use of visualization in the strategic-planning process. Long Range Planning, 42(1), 42-74.

Holloway, J. (2016). What stakeholder management should learn from sales and marketing? Darren, Dalcher (org). Further Advances in Project Management: Guided Exploration in Unfamiliar Landscapes (1 ${ }^{\text {st }}$ Ed). New York: Gower Book. p. 236.

Kerzner, H. (2011). Gerenciamento de Projetos-Uma Abordagem Sistêmica para Planejamento. Programação e Controle (10. Ed), São Paulo: Blucher. 
Larkin, J. H., \& Simon, H. A. (1987). Why a diagram is (sometimes) worth ten thousand words. Cognitive science, 11(1), 65-100.

Lester, A. (2017). Project management, planning and control: managing engineering, construction and manufacturing projects to PMI, APM and BSI standards. ( $7^{\mathrm{a}} \mathrm{ed}$.) Cambridge: Elsevier.

Martins Jr., S. L. (2016). Efetividade Do Gerenciamento De Projetos Utilizando Ferramentas Da Web 2.0 E Gestão Do Conhecimento. Projetos e Dissertações em Sistemas de Informação e Gestão do Conhecimento, 4(2).

McNely, B. J. (2013, September). Visual research methods and communication design. In Proceedings of the 31 st ACM international conference on Design of communication (pp. 123-132). ACM.

Molena, A., Rovai, R. L., Paes da Rosa, C. D., \& Plonsk, G. A. (2017). Comunicação Em Projetos: Um Estudo De Levantamento Desse Fator Crítico De Sucesso. Gestão \& Planejamento-G\&P, 18.

Monecke, A. \& Leisch, F. (2012). semPLS: structural equation modeling using partial least squares. Journal of Statistical Software, 48 (3), 1-32.

Patterson, R. E., Blaha, L. M., Grinstein, G. G., Liggett, K. K., Kaveney, D. E., Sheldon, K. C., \& Moore, J. A. (2014). A human cognition framework for information visualization. Computers \& Graphics, 42 (Special), 42-58.

Pires, H. F. (2008). Governança Global da Internet: A representação de topônimos de países no ciberespaço. Diez años de cambios en el Mundo, en la Geografía y en las Ciencias Sociales, 1999-2008. Actas del X Coloquio Internacional de Geocrítica, 26-30.

PMI - Project Management Institute. (2013) A Guide to the Project Management Body of Knowledge (PMBOK® Guide) (5th ed.). Pennsylvania: Project Management Institute, Inc.

Radujković, M., \& Sjekavica, M. (2017). Project Management Success Factors. Procedia Engineering, 196 (1), 607-615.
Reddivari, S. (2013, July). Visual analytics for software requirements engineering. In Requirements Engineering Conference (RE), 2013 21st IEEE International (pp. 389-392). IEEE.

Rocha, T., \& Goldschmidt, A. (2010). Gestão dos stakeholders. São Paulo: Saraiva.

Rose, G. (2014). On the relation between 'visual research methods' and contemporary visual culture. The Sociological Review, 62(1), 24-46.

Schneider, R. M., Barbosa, J. G. P., Bouzada, M. A. C., \& Gonçalves, A. A. (2016). A influência de fatores organizacionais na gestão de projetos de tecnologia da informação (TI). Revista Gestão \& Tecnologia, 16(1), 157-183.

Sibbet, D. (2013). Reuniões visuais: como gráficos, lembretes autoadesivos e mapeamento de ideias podem transformar a produtividade de um grupo. Rio de Janeiro: Alta Books.

Sindiy, O., Litomisky, K., Davidoff, S., \& Dekens, F. (2013). Introduction to information visualization (infovis) techniques for model-based systems engineering. Procedia Computer Science, 16(1), 4958 .

Teixeira, J., Schoenardie, R., Garcia, L., Merino, E., \& Paladini, E. (2012). Gestão visual: uma proposta de modelo para facilitar o processo de desenvolvimento de produtos. In Conferência Nacional de Integração do Design, Engenharia e Gestão para Inovação (Vol. 2, pp. 1-9).

Van den Ende, L., \& Van Marrewijk, A. (2014). The ritualization of transitions in the project life cycle: A study of transition rituals in construction projects. International Journal of Project Management, 32(7), 1134-1145.

Vargas, R. V. (2016). Gerenciamento de Projetos $8 a$ ed.: Estabelecendo diferenciais competitivos. Rio de Janeiro: Brasport.

Vergara, S. C. (2015). Métodos de pesquisa em administração. 6. ed. São Paulo: Atlas. 